\title{
Physical Inactivity and COVID-19: When Pandemics Collide
}

\author{
Andrea Ramirez Varela, Robert Sallis, Alex V. Rowlands, and James F. Sallis
}

The 3 new Lancet series papers on physical activity ${ }^{1-3}$ build on the evidence presented in 2 previous series ${ }^{4,5}$ that underlined the pandemic magnitude of physical inactivity, its health, social, environmental and economic global consequences, and the urgency to take immediate multisectoral actions to make active living an affordable and accessible choice for all population groups. These messages were based on unquestionable evidence of the beneficial effects of physical activity on virtually every system of the body, supporting it as a "best buy in public health."6 Increasing physical activity could save 5.3 million lives per year, ${ }^{4}$ and it is critical for the prevention and control of multiple noncommunicable diseases (NCDs) that account for $74 \%$ of deaths globally. ${ }^{7}$ However, an estimated 1 in 3 adults and 3 in 4 adolescents worldwide did not meet the physical activity guidelines before the pandemic. ${ }^{8,9}$ Now in 2021, the failure to take seriously evidence-based recommendations to increase physical activity has contributed to the estimated 4.2 million COVID-19 deaths worldwide. ${ }^{10}$ It is clear that the inactivity pandemic is foundational in the global explosion of NCDs that have collided so tragically with the COVID-19 pandemic. ${ }^{11}$

The COVID-19 pandemic has generated more interest in the little known benefits of physical activity for infectious diseases, including reduced risk of infection, enhanced immune system function to prevent and hasten recovery from infections, and increased effectiveness of vaccination. ${ }^{12,13}$ Evidence is also emerging of physical activity benefits specific to severe COVID-19 outcomes, defined by hospitalization, intensive care unit admission, or death. A study was conducted in over 48,000 COVID-19 patients who had multiple physical activity measures prior to diagnosis. After adjusting for demographics and chronic diseases, compared with those meeting physical activity guidelines, those who were inactive were 2.26 times more likely to be admitted to the hospital, 1.73 times more likely to need intensive care unit care, and 2.49 times more likely to die. ${ }^{13}$ Other than advanced age and history of organ transplant, physical inactivity was the biggest risk factor for severe COVID-19 outcomes. Other studies have shown similar associations. ${ }^{14,15}$

Partly because evidence-based interventions (eg, behavior change training) and national policies (eg, provide safe facilities for walking and bicycling $)^{7}$ to promote physical activity were not sufficiently implemented before or during the COVID-19

Ramirez Varela is with the School of Medicine, Universidad de los Andes, Bogota, Colombia. R. Sallis is with Department of Family and Sports Medicine, Kaiser Permanente Medical Center, Fontana, CA, USA. Rowlands is with the Diabetes Research Centre, Leicester Diabetes Centre, Leicester General Hospital Gwendolen Rd, Leicester, United Kingdom; and the National Institute for Health Research, Leicester Biomedical Research Centre, Leicester General Hospital, Leicester, United Kingdom. J.F. Sallis is with the Herbert Wertheim School of Public Health and Human Longevity Science, University of California San Diego, La Jolla, CA, USA; and the Mary MacKillop Institute for Health Research, Australian Catholic University, Melbourne, Australia. Ramirez Varela (an-rami2@uniandes.edu.co) is corresponding author. pandemic, ${ }^{16}$ there is evidence the pandemic itself (and various restrictions to limit spread of the virus) had detrimental impacts on physical activity globally. ${ }^{17,18}$ Despite highly variable measurement methods, most studies described a significant decrease in self-reported or objectively assessed physical activity, along with increased sedentary behavior (sitting), regardless of age, health status, or geographic location, when comparing pre-COVID-19 periods with COVID-19 lockdowns and/or post-COVID-19 periods. This decrease in activity is particularly concerning in those already at risk of poor health outcomes, including people with disabilities, previous NCDs, poor mental health, and those who are already inactive or obese. ${ }^{17,18}$

The long-term health effects of this worldwide decrease in physical activity remain to be determined, but it is clear that public health leaders missed key opportunities to act on the extensive evidence of physical activity benefits before and during the pandemic. Limited attention to promoting physical activity might have been due to low awareness of its benefits for infectious diseases, ${ }^{6}$ as well as the lack of direct evidence of benefits for COVID-19 outcomes. However, evidence about the benefits of physical activity for NCDs and mental health have been well known for decades and should not have been ignored. Pandemic-related anxiety and stress continue to affect the global population, ${ }^{19}$ and it was evident from early in the pandemic that most deaths occurred among those with preexisting NCDs. ${ }^{13}$

While the World Health Organization and some countries acknowledged inactivity as a risk factor for severe COVID-19, many countries did not. Experts anticipate that COVID-19 will likely become endemic and other infectious diseases will emerge. ${ }^{20}$ Therefore, we propose high priorities for research and public health action to promote physical activity. Physical activity should be considered a critical component of pandemic control measures, and physical activity experts should be part of infectious disease response teams. Because physical activity has been shown to enhance the efficacy of vaccines, ${ }^{12}$ funding should be directed to investigate whether it might prolong the efficacy of the COVID-19 vaccines and impact effectiveness against new strains of the virus.

Physical activity creates a healthier population ${ }^{4}$ that is more resilient to infection ${ }^{12}$ and less likely to develop severe COVID-19 outcomes. ${ }^{13}$ Therefore, updating public health messaging to reflect evidence of physical activity's role in protecting against infectious diseases as well as NCDs is critical. Children and adolescents, the elderly, and people with disabilities should be specifically targeted for intervention strategies, given the high prevalence of inactivity in these groups and potential for numerous physical and mental health benefits. ${ }^{2,3}$

The COVID-19 pandemic continues to advance in many countries, and it will likely take many months before most of the world's population is vaccinated. Urgent action is needed to tackle the collision of the inactivity, NCD, and COVID-19 pandemics (also known as a syndemic), including effective public health strategies to promote physical activity as a means of reducing health inequities, 
morbidity, and mortality from COVID-19 and NCDs. ${ }^{11,13}$ Therefore, we recommend that global action and collaboration across multiple sectors of society, guided by the World Health Organization Global Action Plan for Physical Activity, be implemented immediately around the globe. ${ }^{7}$

Let us learn from past mistakes and not continue to ignore physical activity as an important pandemic control measure. With future pandemics looming, a failure to act now should be considered public health malpractice.

\section{Acknowledgments}

J.F.S. received royalties from Gopher Sport Inc and San Diego State University Research Foundation for SPARK youth physical activity programs that are licensed through SDSURF to Gopher Sport Inc; consulting fees from Gopher Sport Inc; and is an unpaid member of the Board of Directors of Rails to Trails Conservancy, all unrelated to the topic of this Comment. A.R.V., R.S., and A.V.R. declare no competing interests.

\section{References}

1. Bauman A, Kamada M, Reis R, et al. An evidence-based assessment of the impact of the Olympic games on population levels of physical activity. Lancet. 2021;398(10298):456-464. doi:10.1016/S01406736(21)01165-x

2. Martin Ginis K, van der Ploeg H, Foster C, et al. Physical activity among people with disabilities: a global perspective. Lancet. 2021;398(10298):443-455. doi:10.1016/S0140-6736(21)01164-8

3. van Sluijs E, Ekelund U, Crochemore-Silva I, et al. Physical activity behaviours in adolescence: current evidence and opportunities for intervention. Lancet. 2021;398(10298):429-442. doi:10.1016/ S0140-6736(21)01259-9

4. Lee IM, Shiroma EJ, Lobelo F, Puska P, Blair SN, Katzmarzyk PT. Effect of physical inactivity on major non-communicable diseases worldwide: an analysis of burden of disease and life expectancy. Lancet. 2012;380(9838):219-229. doi:10.1016/S0140-6736(12) 61031-9

5. Kohl HW III, Craig CL, Lambert EV, et al. The pandemic of physical inactivity: global action for public health. Lancet. 2012;380(9838): 294-305. doi:10.1016/S0140-6736(12)60898-8

6. Das P, Horton R. Physical activity-time to take it seriously and regularly. Lancet. 2016;388(10051):1254-1255. doi:10.1016/S01406736(16)31070-4

7. World Health Organization (WHO). Global Action Plan on Physical Activity 2018-2030: More Active People for a Healthier World. Geneva: World Health Organization. 2018.

8. Guthold R, Stevens GA, Riley LM, Bull FC. Worldwide trends in insufficient physical activity from 2001 to 2016: a pooled analysis of
358 population-based surveys with 1.9 million participants. Lancet Glob Health. 2018;6(10):e1077-e1086. PubMed ID: 30193830 doi:10.1016/S2214-109X30357-7

9. Guthold R, Stevens GA, Riley LM, Bull FC. Global trends in insufficient physical activity among adolescents: a pooled analysis of 298 population-based surveys with 1. 6 million participants. Lancet Child Adolesc Health. 2020;421:23-35. doi:10.1016/ S2352-4642(19)30323-2

10. Johns Hopkins University. COVID-19 Map - Johns Hopkins Coronavirus Resource Center. 2021. https://coronavirus.jhu.edu/map.html

11. Horton R. Offline: COVID-19 is not a pandemic. Lancet. 2020; 396(10255):874. doi:10.1016/S0140-6736(20)32000-6

12. Chastin SFM, Abaraogu U, Bourgois JG, et al. Effects of regular physical activity on the immune system, vaccination and risk of community-acquired infectious disease in the general population: systematic review and meta-analysis. Sports Med. 2021:51(8):16371686. PubMed ID: 33877614 doi:10.1007/s40279-021-01466-1

13. Sallis R, Young DR, Tartof SY, et al. Physical inactivity is associated with a higher risk for severe COVID-19 outcomes: a study in 48440 adult patients. Br J Sports Med. 2021. doi:10.1136/bjsports-2021104080

14. Rowlands AV, Kloecker DE, Chudasama Y, et al. Association of timing and balance of physical activity and rest/sleep with risk of COVID-19: a UK Biobank Study. Mayo Clin Proc. 2021:96(1):156164. doi:10.1016/j.mayocp.2020.10.032

15. Lobelo F, Bienvenida A, Leung S, et al. Clinical, behavioural and social factors associated with racial disparities in COVID-19 patients from an integrated healthcare system in Georgia: a retrospective cohort study. BMJ Open. 2021;11(5):e044052. doi:10.1136/bmjopen-2020044052

16. Pratt M, Varela AR, Salvo D, Kohl HW III, Ding D. Attacking the pandemic of physical inactivity: what is holding us back? Br J Sports Med. 2020;54(13):760-762. PubMed ID: 31704698 doi:10.1136/ bjsports-2019-101392

17. McCarthy H, Potts HW, Fisher A. Physical activity behavior before, during, and after COVID-19 restrictions: longitudinal smartphonetracking study of adults in the United Kingdom. J Med Internet Res. 2021;23(2):e23701. PubMed ID: 33347421 doi:10.2196/23701

18. Stockwell S, Trott M, Tully M, et al. Changes in physical activity and sedentary behaviours from before to during the COVID-19 pandemic lockdown: a systematic review. BMJ Open Sport Exerc Med. 2021;721:e000960. doi:10.1136/bmjsem-2020-000960

19. Pierce M, Hope H, Ford T, et al. Mental health before and during the COVID-19 pandemic: a longitudinal probability sample survey of the UK population. Lancet Psychiatry. 2020;7(10):883-892. PubMed ID: 32707037 doi:10.1016/S2215-0366(20)30308-4

20. Phillips N. The coronavirus is here to stay-here's what that means. Nature. 2021;590(7846):382-384. PubMed ID: 33594289 doi:10. 1038/d41586-021-00396-2 